SMALL INTESTINE

\title{
Increased duodenal iron uptake and transfer in a rat model of chronic hypoxia is accompanied by reduced hepcidin expression
}

\author{
P S Leung, S K Srai, M Mascarenhas, L J Churchill, E S Debnam
}

See end of article for

Gut 2005;54:1391-1395. doi: 10.1136/gut.2004.062083

authors' affiliations

Correspondence to: Dr E S Debnam, Department of Physiology, Royal Free and University College School of Medicine, University College London (Hampstead Campus), Rowland Hill St, London NW3 2PF, UK: e.debnam@medsch. ucl.ac.uk

Revised version received 20 April 2005 Accepted for publication 26 April 2005 Published online first 24 May 2005 ....................
Background: Despite the requirement for increased iron delivery for erythropoiesis during hypoxia, there is very little information on how duodenal iron uptake and its transfer to the blood adapts to this condition. Aims: To assess the effects of 30 days of chronic hypoxia in rats on luminal iron uptake and transfer of the metal to blood, together with gene expression of hepcidin, a proposed negative regulator of iron transport. Methods: $59-\mathrm{Fe}$ uptake by isolated duodenum and its transfer to blood by in vivo duodenal segments was measured after exposure of rats to room air or $10 \%$ oxygen for four weeks. Liver hepcidin expression was measured by real time reverse transcription-polymerase chain reaction. The effects of hypoxia on hepcidin gene expression by HepG2 cells was also determined.

Results: Hypoxia did not affect villus length but enhanced (+192.6\%) luminal iron uptake by increasing the rate of uptake by all enterocytes, particularly those on the upper villus. Hypoxia promoted iron transfer to the blood but reduced mucosal iron accumulation in vivo by $66.7 \%$. Hypoxia reduced expression of hepcidin mRNA in both rat liver and HepG2 cells.

Conclusions: Prolonged hypoxia enhances iron transport from duodenal lumen to blood but the process is unable to fully meet the iron requirement for increased erythropoiesis. Reduced secretion of hepcidin may be pivotal to the changes in iron absorption. The processes responsible for suppression of hepcidin expression are unknown but are likely to involve a direct effect of hypoxia on hepatocytes.
$\mathrm{C}$ ontrol of intestinal iron uptake is necessary to maintain body iron status as there is no established route for regulating iron excretion. The increased rate of erythropoiesis that occurs during hypoxia requires a greater supply of iron for haem synthesis, and increased intestinal iron uptake occurs in the adaptation of humans to high altitude. ${ }^{1}$ Animal studies have shown that three days of hypoxia stimulates duodenal iron absorption by processes involving an increased rate of iron uptake across the brush border membrane..$^{2-4}$ The systemic signals that trigger the intestinal response to hypoxia are unknown. However, there is growing evidence that the liver derived peptide hepcidin, originally identified as an antimicrobial peptide, functions as a negative regulator of duodenal iron absorption and is released in response to the level of liver iron. ${ }^{5-8}$ Recent findings that treatment of mice with synthetic hepcidin decreased iron transfer to the blood, ${ }^{9}$ and that the peptide rapidly reduced iron uptake in an intestinal cell line, ${ }^{10}$ further suggest a key role for the peptide in the regulation of intestinal iron uptake. Our present work has, for the first time, used an animal model of chronic hypoxia, equivalent to the partial pressure of inspired oxygen at an altitude of $5000 \mathrm{~m}$ above sea level, to study changes in iron uptake from the duodenal lumen and its transfer to blood. We have also assessed the involvement of hepcidin in the response to hypoxia by measuring levels of hepcidin mRNA in rat liver and in a human hepatoma derived cell line before and after direct exposure to reduced oxygen.

\section{METHODS}

Iron uptake across rat duodenum

Sprague-Dawley rats (initial weight 90-100 g), supplied by the Animal Services Centre of the Chinese University of Hong Kong, were exposed for four weeks to room air mixed with nitrogen, to reduce oxygen concentration to $10 \%$, or room air alone. ${ }^{11}$ Briefly, cages containing four rats were kept in sealed Perspex chambers with inflow and outflow ports for inspired normal or hypoxic air. Water and rat chow were supplied ad libitum to the hypoxic group and the weight of chow consumed was closely monitored so that the same amount of chow was also supplied to the normoxic group. Animals were weighed twice weekly. All experimental procedures were approved by the Animal Experimentation Ethics Committee of the Chinese University of Hong Kong.

At the end of the four week period, animals were anaesthetised with pentobarbitone sodium $(90 \mathrm{mg} / \mathrm{kg}$ intraperitoneally) and blood samples were removed by cardiac puncture and sent to a commercial laboratory (Hong Ning Xray and Laboratory Company Ltd, Kowloon, Hong Kong) for determination of haemoglobin level, haematocrit, and red blood cell count. Liver samples were removed for determination of hepcidin mRNA expression and these were snap frozen in liquid nitrogen. For mucosal iron uptake experiments, $3 \mathrm{~cm}$ of isolated duodenum were everted, mounted on a glass rod, and preincubated for five minutes at $37^{\circ} \mathrm{C}$ in HEPES buffer containing $10 \mathrm{mM}$ glucose. Tissue was then exposed for five minutes to preincubation buffer with $0.2 \mathrm{mM}$ 59- $\mathrm{Fe}^{2+}$ (Perkin Elmer, Boston, Massachusetts, USA, final specific activity $9.25 \mathrm{MBq} / \mu \mathrm{mol}$ ) complexed with $4 \mathrm{mM}$ ascorbate (final buffer $\mathrm{pH}$ 6.5). The tissue was then washed free of surface bound iron using a solution containing $2 \mathrm{mM}$ unlabelled iron, fixed in formaldehyde-saline, and weighed. The 59-Fe activity of tissues was determined by gamma counting (Packard 5003; Cobra II Auto-Gamma,

Abbreviations: PCR, polymerase chain reaction; GAPDH, glyceraldehyde-3-phosphate dehydrogenase; HPRT, hypoxanthine phosphoribosyl transferase; Epo, erythropoietin 
Global Medical Instrumentation, Inc., Minnesota, USA) and were then processed for histology and autoradiography. ${ }^{4}$ Grain counts of developed autoradiographs were carried out using a full width $50 \mu \mathrm{m}$ area moving from the villus base to the tip.

Other animals were used to measure the rate of transfer of $59-\mathrm{Fe}$ from the duodenal lumen to blood. ${ }^{12}$ Rats were anaesthetised as described above and the duodenum, $0.5 \mathrm{~cm}$ from the stomach to the ligament of Treitz, was washed through with warm $0.15 \mathrm{M} \mathrm{NaCl}$ followed by air. The lower end was tied off and $1 \mathrm{ml}$ of uptake buffer (see above) was instilled proximally from a tied-in syringe. Blood samples $(0.5 \mathrm{ml})$ were removed via a carotid cannula at 10 , 20, and 30 minutes, during which time animal body temperature was maintained at $37^{\circ} \mathrm{C}$. The duodenal segment was then removed from the animal, its lumen flushed thoroughly with buffer containing $2 \mathrm{mM}$ unlabelled iron, followed by $0.15 \mathrm{M} \mathrm{NaCl}$, and the length was measured. The mucosal layer was scraped off and weighed and, together with weighed blood samples, was gamma counted for determination of 59-Fe activity.

\section{Hepcidin mRNA}

Frozen rat liver sections were ground to powder using a pestle and mortar. RNA isolation was performed using Qiagen RNAeasy kit (Qiagen, Sussex, UK), and $1 \mu \mathrm{g}$ of total RNA was used for cDNA synthesis with the Abgene Reverse-iT 1st Strand Synthesis Kit (ABgene, Surrey, UK). RNA concentration and purity were determined by spectrophotometry. The resulting CDNA transcripts of liver mRNA were used for real time polymerase chain reaction (PCR) amplification using the Roche Lightcycler (Roche Diagnostics, Germany) and QuantiTect SYBR Green PCR kit (Qiagen), according to the manufacturer's protocol. Specific primers were designed from the rat sequences for hepcidin and the constitutively expressed gene actin as follows: hepcidin: forward CAC GAG GGC AGG ACA GAA GGC AAG, reverse CAA GGT CAT TGC TGG GGT AGG ACA G; actin: forward GAC GGC CAA GTC ATC ACT ATT, reverse CCA CAG GAT TCC ATA CCC AAG A.

To quantify hepcidin mRNA expression, standard curves were generated with known amounts of each gene product. A ratio of relative abundance of the hepcidin gene to actin was calculated by the Lightcycler Relative Quantification software version 1.0 (Roche Diagnostics). Melting curve analysis was carried out to ensure primer specificity. PCR products were also analysed by gel electrophoresis and visualised using a Bio-Rad multi-imager (Bio-Rad, Herts, UK).

\section{Hypoxic exposure of HepG2 cells}

HepG2 cells were cultured in RPMI1640 medium (Sigma, St Louis, Missouri, USA) containing L-glutamine (Sigma), antibiotics, and $10 \%$ fetal calf serum (Sigma) and maintained in $5 \% \mathrm{CO} 2$ at $37^{\circ} \mathrm{C}$. Cells were seeded in $5 \mathrm{~cm}^{2}$ wells until confluent. Cells were then subjected to hypoxia by placing them in stainless steel chambers as described previously. ${ }^{13}$ The chambers were evacuated until the desired oxygen

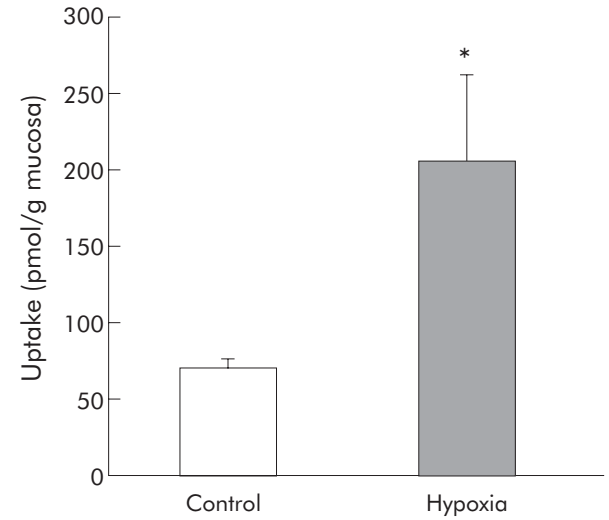

Figure 1 Effects of hypoxia on duodenal iron uptake. $59-\mathrm{Fe}^{2+}$ uptake by isolated everted duodenum after incubation of the tissue in iron containing buffer for five minutes. Iron was present in the uptake buffer at a concentration of $0.2 \mathrm{mM}$. Data are means (SEM); $\mathrm{n}=6$ and 7 for control and hypoxia respectively. ${ }^{*} \mathrm{p}<0.05$.

partial pressure was reached, deduced by measuring the pressure with an electronic pressure sensor (SMC digital pressure sensor; SMC Corporation, Tokyo, Japan). The chambers were then refilled with a gas mixture containing $5 \% \mathrm{CO}_{2}$ and $95 \% \mathrm{~N}_{2}$. A final oxygen concentration of $1 \%$ was used. The chambers were then placed in an incubator at $37^{\circ} \mathrm{C}$. After 24 or 48 hours, cells were used for determination of hepcidin mRNA. All procedures for RNA isolation, cDNA synthesis, PCR amplification, and quantification of human hepcidin mRNA expression were performed as above for rat hepcidin mRNA. The primers used for HepG2 cDNA studies were designed for the sequences of human hepcidin and the housekeeping genes glyceraldehyde-3-phosphate dehydrogenase (GAPDH) and hypoxanthine phosphoribosyl transferase (HPRT) as follows: hepcidin: forward CTG CAA CCC CAG GAC AGA G, reverse GGA ATA AAT AAG GAA GGG AGG GG; GAPDH: forward TGG TAT CGT GGA AGG ACT C, reverse AGT AGA GGC AGG GAT GAT G; HPRT: forward TTG TAG CCC TCT GTG TGC TCA AG, reverse GCC TGA CCA AGG AAA GCA AAG TC.

\section{Statistics}

Values are given as means (SEM). Statistical significance was determined by an unpaired Student's $t$ test, with $\mathrm{p}<0.05$ taken as significant.

\section{RESULTS}

Four weeks of hypoxia was without effect on animal body weight but caused significant increases in blood haemoglobin concentration, haematocrit, and erythrocyte count (table 1).

Hypoxia enhanced significantly $(+192.6 \%)$ the rapid unidirectional uptake of 59-Fe across the brush border membrane of isolated duodenum (fig 1). Autoradiographic analysis of this tissue revealed silver grains overlying the villus (fig 2). The expected pattern of lower rates of uptake at

Table 1 Effects of hypoxia on final body weight, haemoglobin concentration, haematocrit, and red blood cell count. Rats were exposed for 30 days to air containing $10 \%$ oxygen or room air

\begin{tabular}{|c|c|c|c|c|}
\hline & Final body weight (g) & Haemoglobin (g/dl) & Haematocrit & $\begin{array}{l}\text { Red blood cell count } \\
\left(\times 10^{12} / \mathrm{I}\right)\end{array}$ \\
\hline $\begin{array}{l}\text { Control } \\
\text { Hypoxic }\end{array}$ & $\begin{array}{l}210.4(5.6)(n=18) \\
223.6(6.2)(n=21)\end{array}$ & $\begin{array}{l}12.72(0.41)(n=5) \\
18.71(0.59)(n=7)^{* *}\end{array}$ & $\begin{array}{l}0.41(0.01)(n=5) \\
0.60(0.02)(n=7)^{* *}\end{array}$ & $\begin{array}{l}7.33(0.48)(n=5) \\
9.43(0.18)(n=7)^{* *}\end{array}$ \\
\hline
\end{tabular}



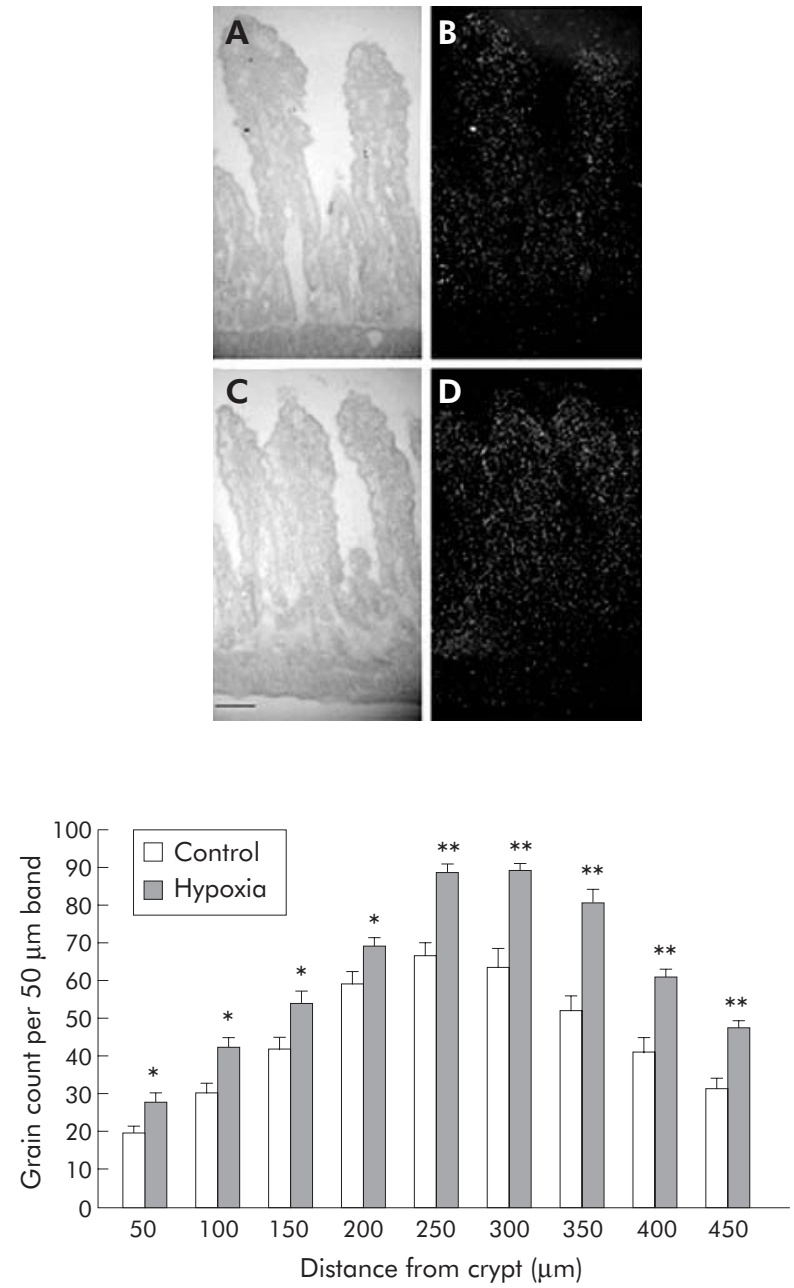

Figure 2 Autoradiography of iron uptake. Autoradiographs (upper panel) show rat duodenal villi in tissue from control (A, B) and hypoxic (C, D) rats. Intestinal sections were mounted on slides, dipped in photographic emulsion, and developed one day later. The underlying tissue was stained with Light Green. Silver grains representing iron uptake were most clearly seen using dark field photography (B, D). Light fields $(A, C)$ showed underlying tissue histology. The lower panel shows the positional dependence of $59-\mathrm{Fe}^{2+}$ uptake by villus attached enterocytes under control and hypoxic conditions. Data were obtained from 18 villi (three villi from each of six control or hypoxic rats). Values are means (SEM). ${ }^{*} p<0.05,{ }^{* *} p<0.001$. Scale bar $=50 \mu \mathrm{m}$.

the base and tip of the villus, due to increasing cell maturation and apoptosis, respectively, and maximal uptake in the mid-villus region (fig 2) was seen. Hypoxia had no effect on this pattern of iron uptake but increased significantly the rate of uptake into enterocytes along the entire villus length, the differences being more significant at the upper region. Villus length was unaffected by hypoxia (mean (SEM): control 602.8 (26.3); hypoxic 550.9 (11.5) $\mu \mathrm{m}$ ).

Hypoxia promoted the rate of appearance of 59-Fe in blood at 20 and 30 minutes after instillation of buffer containing the radionucleotide into the duodenal lumen (fig 3). After removal of the 30 minute blood sample, the duodenum was isolated and its mucosa was removed and gamma counted. 59-Fe accumulation in the exposed duodenal mucosa was significantly reduced by hypoxia (mean (SEM): control 0.81 (0.20); hypoxic 0.27 (0.06) pmol/mg wet weight mucosa; $\mathrm{p}<0.05, \mathrm{n}=8$ per group).

Increased iron uptake by isolated tissue and in vivo during hypoxia was accompanied by reduced hepatic expression of

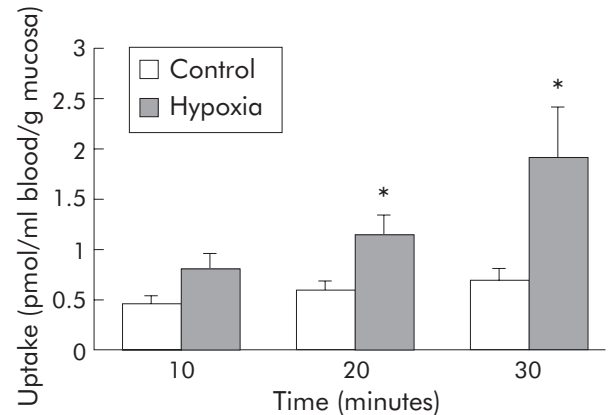

Figure 3 Time course of $59-\mathrm{Fe}^{2+}$ appearance in blood after instillation of buffer containing $59-\mathrm{Fe}^{2+}$ into closed duodenal loops under control and hypoxic conditions. Iron was present in the uptake buffer at a concentration of $0.2 \mathrm{mM}$. Data are means (SEM); $n=8$ per group. ${ }^{*} \mathrm{p}<0.05$ compared with control values.

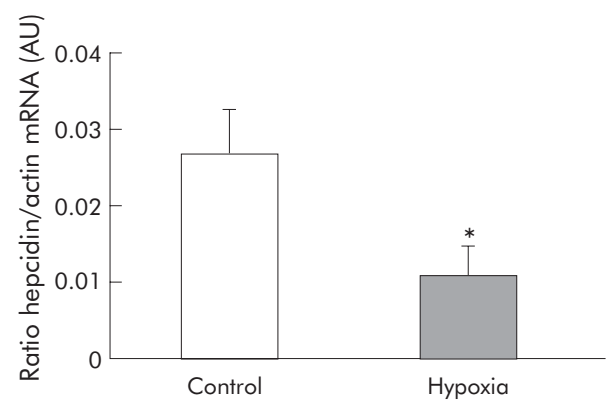

Figure 4 Effects of hypoxia on expression of liver hepcidin mRNA. Real time polymerase chain reaction analysis of total RNA isolated from liver samples removed from control and hypoxic rats. Data were normalised to levels of actin, expressed as arbitrary units (AU). Means (SEM) of liver samples from each of three (control) and four (hypoxia) animals. ${ }^{*} \mathrm{p}<0.01$.

hepcidin mRNA (fig 4). Hypoxia had no effect on expression of the housekeeping gene actin (data not given).

To examine whether the reduced hepcidin mRNA was due to a direct effect of hypoxia on rat liver, levels of hepcidin mRNA of HepG2 cells were determined after exposing them to $1 \%$ oxygen for up to 48 hours. Results showed that expression of hepcidin mRNA, when compared with the housekeeping gene GAPDH, was reduced by $24.8 \%$ and $50.0 \%$ after 24 and 48 hours of hypoxia, respectively, the value at 48 hours being significantly different to the control value (fig 5). This action of hypoxia was not due to altered expression of the glycolytic enzyme GAPDH as GAPDH/HPRT

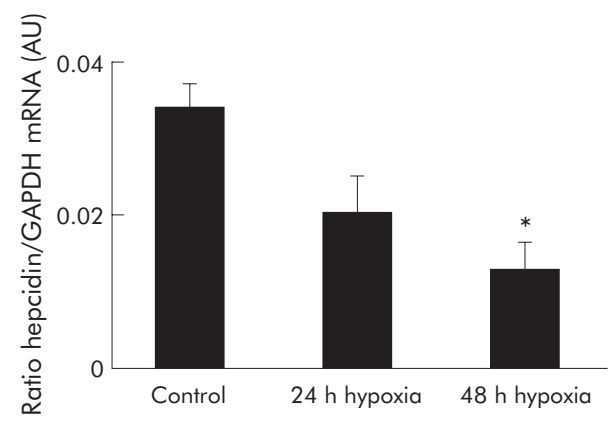

Figure 5 Effects of 24 or 48 hours of hypoxia on expression of hepcidin mRNA in HepG2 cells, normalised to glyceraldehyde-3phosphate dehydrogenase (GAPDH), expressed as arbitrary units (AU). Means (SEM) of samples from each of three (control and 24 hour hypoxia) and four (48 hour hypoxia) cells, ${ }^{*} \mathrm{p}<0.02$. 
ratios were unaffected by hypoxia (control: 4.02 (0.45), $\mathrm{p}>0.5$; 24 hour hypoxia: 2.57 (0.38), $\mathrm{p}>0.5 ; 48$ hour hypoxia: $3.68(0.49), \mathrm{p}>0.5)$.

\section{DISCUSSION}

The erythropoietic demands imposed by chronic hypoxia require a supply of iron to the erythroid marrow that cannot be achieved by available body stores of the metal. Our study provides the first detailed analysis of the effects of chronic hypoxia on duodenal iron transport and shows that increased entry of iron into the body during hypoxia occurs by both stimulation of iron entry across luminal membrane into the enterocyte and enhanced iron transfer across the basolateral membrane into the perfusing blood. These changes are independent of villus length, implying altered function of individual enterocytes. Our previous studies on the response to acute hypoxia make it likely that increased iron movement across the brush border is due in part to an increased electrical driving force for proton mediated iron transport across this membrane. ${ }^{12}$ An important observation in our present study is that, despite higher iron uptake by isolated duodenum, we found significantly lower levels of absorbed iron in hypoxic mucosa following the in vivo transfer study. This implies a proportionally greater effect of hypoxia on iron transfer across the basolateral membrane to the blood.

There is growing evidence that intestinal iron transport is under hormonal control. Early studies suggested a humoral effect on iron transport that was linked to an inflammatory response. ${ }^{14}$ Hepcidin has since been identified as an antimicrobial peptide synthesised in the liver ${ }^{15}{ }^{16}$ that has a central role in body iron homeostasis by acting as a negative regulator of duodenal iron transport. The strength of the evidence linking this 20-25 amino acid peptide to iron transport is now so great that it has acquired the status of a regulatory peptide. For example, there is a reciprocal relationship between the rate of iron transport and expression of hepcidin in a variety of clinical and experimental conditions, ${ }^{717}$ and work using knockout mice have shown that deletion of the hepcidin gene results in body iron overload. ${ }^{17}$ Our recent studies showing that treatment with hepcidin decreases iron transport in mouse duodenum, ${ }^{9}$ and an intestinal cell line, ${ }^{10}$ supports the conclusion of many others that the peptide has a direct inhibitory effect on enterocyte iron transport. ${ }^{5-8} 17-19$

It is likely that the increased iron transport seen in our present study is due, at least in part, to reduced expression and secretion of hepcidin, perhaps as a consequence of a depleted level of liver iron following mobilisation of the metal from the liver to erythroid marrow. The fact that increased iron uptake occurred after a four week period of hypoxia is significant in that it indicates a continuing high iron demand despite a $47 \%$ increased blood haemoglobin level. The only other study of the effects of hypoxia on hepcidin expression reported consistently decreased expression in mice after four days of hypoxia but equivocal data after 12 days. ${ }^{5}$

We further studied the effects of hypoxia on hepcidin expression using human hepatoma derived HepG2 cells. These were chosen for the study as they have pathways for obtaining iron from transferrin ${ }^{20}$ and hence are suitable for studying the hepatic control of iron metabolism. Our data imply that at least part of the response to hypoxia in rats is due to a direct action of low oxygen partial pressure on hepatocytes. HepG2 cell experiments used a lower level of oxygen (approximately $7.6 \mathrm{~mm} \mathrm{Hg}$ partial pressure) than that used in the rat study because, even under normal conditions, the partial pressure of oxygen in blood supplying hepatocytes is considerably lower than the value present in systemic blood as a large proportion of hepatic blood has undergone partial deoxygenation during its passage through the mesenteric circulation. The level of oxygen in blood supplying hepatocytes in rats breathing normal or $10 \%$ oxygen is unknown. Whether the direct action of hypoxia on hepcidin expression involves the binding of the transcription factor HIF-1 to a hypoxia response element on hepcidin mRNA is unclear at the present time. However, the evidence suggests that HIF-l is involved in most, if not all, of the cellular response to hypoxia. ${ }^{21}$

With regard to the action of hepcidin on enterocyte iron transport, studies show that the peptide downregulates expression of both DMTl and DcytB at the brush border and has either no effect ${ }^{9}{ }^{10}$ or decreases ${ }^{72}$ basolateral iron transfer and expression of Iregl, the iron transporter at this membrane. It is clear that the effect of hepcidin on iron exit from the enterocyte is complex and that the peptide may act in concert with other regulatory factors, particularly in response to chronic effects on iron transport. Hypoxia stimulates the secretion of erythropoietin (Epo) and preliminary studies have shown that Epo promotes iron uptake in an intestinal cell line. ${ }^{23}$ The relation of Epo to increased iron uptake seen in this present work, or to the action of hepcidin, is unknown.

Our autoradiographic findings show that hypoxia stimulates iron uptake by both mature enterocytes at the upper villus as well as newly differentiated cells at the base of the villus. As hepcidin may have a direct action on mature enterocytes, ${ }^{17}$ an important issue to be addressed when a suitable antibody to the hepcidin receptor becomes available is how hypoxia induced changes in transport along the villus relate to the distribution profile of the hepcidin receptor.

In conclusion, our demonstration of stimulatory effects of chronic hypoxia on iron uptake from the lumen and its transfer to blood is relevant not only to body iron homeostasis at high altitude but also for any clinical condition that chronically reduces oxygen intake or its delivery to peripheral tissues. Suppression of hepcidin secretion is likely to be relevant to increased iron uptake but the events that culminate in reduced expression of the peptide at the hepatocyte level are unknown but may involve a direct effect of low oxygen via the transcription factor HIF. Recent work suggests that changes in hepcidin synthesis and secretion in response to an altered level of body iron may also involve the hepatic detection of blood transferrin saturation by at least two hepatic transferrin receptors and the HFE protein. ${ }^{17}{ }^{24}$

\section{ACKNOWLEDGEMENTS}

ESD is grateful to the Royal Society for the award of a travel grant. PSL is grateful to the Research Grants Council of Hong Kong for partial support from a Competitive Earmarked Research Grant (Project No CUHK4364/04M). Dr Jill Norman, Department of Nephrology, University College London, kindly provided facilities for the HepG2 cell experiments.

\section{Authors' affiliations}

P S Leung, Department of Physiology, Faculty of Medicine, The Chinese University of Hong Kong, Shatin, Hong Kong, P R China

S K Srai, M Mascarenhas, Department of Biochemistry and Molecular Biology, Royal Free and University College Medical School, London, UK L J Churchill, E S Debnam, Department of Physiology, Royal Free and University College Medical School, London, UK

Conflict of interest: None declared.

\section{REFERENCES}

1 Reynafarie C, Ramos J. Influence of altitude changes on intestinal iron absorption. J Lab Clin Med 1961;57:848-55.

2 Osterloh KR, Simpson RJ, Snape S, et al. Intestinal iron absorption and mucosal transferrin in rats subjected to hypoxia. Blut 1987;55:421-31.

3 Raja KB, Bjarnason I, Simpson RJ, et al. In vitro measurements and adaptive response of $\mathrm{Fe}^{2+}$ uptake by mouse intestine. Cell Biochem Function 1986;5:69-76. 
4 O'Riordan DK, Debnam ES, Sharp PA, et al. Mechanisms involved in increased iron uptake across rat duodenal brush-border membrane during hypoxia. J Physiol 1997;500:379-84.

5 Nicolas G, Chauvet $C$, Viatte $L$, et al. The gene encoding the iron regulatory peptide hepcidin is regulated by anemia, hypoxia, and inflammation. J Clin Invest 2002;110:1037-44.

6 Roetto A, Papanikolaou G, Politou M, et al. Mutant antimicrobial peptide hepcidin is associated with severe juvenile hemochromatosis. Nat Genet 2003:33:21-2

7 Frazer DM, Wilkins SJ, Becker EM, et al. Hepcidin expression inversely correlates with the expression of duodenal iron transporters and iron absorption in rats. Gastroenterology 2002; 123:835-44.

8 Nemeth $\mathrm{E}$, Valore EV, Territo $M$, et al. Hepcidin, a putative mediator of anemia of inflammation, is a type II acute-phase protein. Blood 2003;101:2461-3.

9 Laftah AH, Ramesh B, Simpson RJ, et al. Effect of hepcidin on intestinal iron absorption in mice. Blood 2004; 103:3940-4.

10 Yamaji S, Sharp P, Ramesh B, et al. Inhibition of iron transport across human intestinal epithelial cells by hepcidin. Blood 2004; 104:2178-80.

11 Leung PS, Lam SY, Fung ML. Chronic hypoxia upregulates the expression and function of AT1 receptor in rat carotid body. J Endocrinol 2000;167:517-24.

12 O'Riordan DK, Sharp P, Sykes RM, et al. Cellular mechanisms underlying the increased duodenal iron absorption in rats in response to phenylhydrazineinduced haemolytic anaemia. Eur J Clin Invest 1995;25:722-7.

13 Mazure NM, Chauvet C, Bois-Joyeux B, et al. Repression of alpha-fetoprotein gene expression under hypoxic conditions in human hepatoma cells: characterization of a negative hypoxia response element that mediates opposite effects of hypoxia inducible factor-1 and c-Myc. Cancer Res 2002:62:1158-65.
14 Raja KB, Duane P, Peters TJ. Effects of turpentine-induced inflammation on the hypoxic stimulation of intestinal Fe3+ absorption in mice. Int J Exp Pathol 1990;71:785-9.

15 Krause A, Neitz S, Magert HJ, et al. LEAP-1, a novel highly disulfide-bonded human peptide, exhibits antimicrobial activity. FEBS Lett 2000;480:147-50.

16 Park $\mathrm{CH}$, Valore EV, Waring AJ, et al. Hepcidin, a urinary antimicrobial peptide synthesized in the liver. J Biol Chem 2001;276:7806-10.

17 Frazer DM, Anderson GJ. The orchestration of body iron intake: how and where do enterocytes receive their cues? Blood Cells Mol Dis 2003:30:288-97.

18 Nicolas G, Bennoun M, Devaux I, et al. Lack of hepcidin gene expression and severe tissue iron overload in upstream stimulatory factor 2 (USF2) knockout mice. Proc Natl Acad Sci U S A 2001;98:8780-85.

19 Walker AP, Partridge J, Srai SK, et al. Hepcidin: what every gastroenterologist should know. Gut 2004;53:624-7.

20 Robb AD, Ericsson M, Wessling-Resnick M. Transferrin receptor 2 mediates a biphasic pattern of transferrin uptake associated with ligand delivery to multivesicular bodies. Am J Physiol 2004;287:C1769-75.

21 Wang GL, Semenza GL. General involvement of hypoxia-inducible factor 1 in transcriptional response to hypoxia. Proc Natl Acad Sci U S A 1993;90:4304-8

22 Yeh KY, Yeh M, Glass J. Hepcidin regulation of ferroportin 1 expression in the liver and intestine of the rat. Am J Physiol 2004;286:G385-94.

23 Tennant J, Anderson E, Sharp P. Erythropoietin stimulates iron uptake across the apical membrane of human intestinal Caco-2 cells. J Physiol 2002;539P:SO79

24 Millard KN, Frazer DM, Wilkins SJ, et al. Changes in the expression of intestinal iron transport and hepatic regulatory molecules explain the enhanced iron absorption associated with pregnancy in the rat. Gut 2004;53:655-60.

\title{
EDITOR'S QUIZ: GI SNAPSHOT
}

\begin{abstract}
Answer
From question on page 1369

The diagnosis was Sister Mary nodule/metastatic umbilical deposit. The half body positron emission tomography (PET) examination (fig 1) demonstrated a circumferential area of increased ${ }^{18} \mathrm{~F}$-fluorodeoxyglucose (FDG) uptake (black arrow) in the region of the cardia of the stomach. This corresponded to the site of endoscopic biopsy that revealed adenocarcinoma of the gastro-oesophageal junction. Another focus of hypermetabolic activity was seen in the periumbilical region (white arrow), representing a metastatic unbiblical deposit. In the 16 detector multislice computed tomography (CT) examination (fig 2), this umbilical lesion was shown as a high density nodule (white arrow).

Sister Mary Joseph, the First Assistant in the early days of the Mayo Clinic, noticed umbilical lesions in those with advanced abdominal malignancy. Sir Hamilton Bailey termed these lesions "Sister Mary nodules," in recognition of the initial observer. They represent metastatic umbilical deposits. It is thought that a combination of generous blood supply, proximity to the peritoneum, and abundant embryological ligaments contribute to the high incidence of these deposits. Histological examination of these lesions usually reveals adenocarcinoma, most commonly originating from the stomach, pancreas, ovary, or colon. As we have demonstrated, various modern day cross sectional imaging techniques such as multidetector CT have successfully demonstrated umbilical malignant nodules. These deposits are now also being increasingly imaged using recent nuclear medicine techniques such as FDG PET.
\end{abstract}

doi: 10.1136/gut.2005.068338 